\title{
The association between substance use and risky sexual behaviors among middle school children
}

\author{
Gemechu B. Gerbi - Cheryl G. Davis - Tsegaye Habtemariam • \\ Berhanu Tameru $\cdot$ David Nganwa $\cdot$ Vinaida Robnett
}

Accepted: February 7, 2008/Published online: 22 February 2008

(C) Springer Science+Business Media, LLC 2008

This article has been withdrawn because it did not successfully complete the external review process.

This article has been withdrawn because it did not successfully complete the external review process.

G. B. Gerbi $(\bowtie)$

Center for Computational Epidemiology, Bioinformatics and Risk Analysis (CCEBRA), College of Veterinary Medicine, Nursing \& Allied Health (CVMNAH), Tuskegee University, 104 Williams-Bowie Hall, Tuskegee, AL 36088, USA

e-mail: gbeyene@tuskegee.edu

T. Habtemariam · B. Tameru · D. Nganwa

V. Robnett

Center for Computational Epidemiology, Bioinformatics and Risk Analysis (CCEBRA), Tuskegee University, 104 WilliamsBowie Hall, Tuskegee, AL 36088, USA

G. B. Gerbi · C. G. Davis

Department of Allied Health, College of Veterinary Medicine, Nursing \& Allied Health (CVMNAH), Tuskegee University,

Tuskegee, AL 36088, USA 